\title{
Salivary microbiomes of indigenous Tsimane mothers and infants are distinct despite frequent premastication
}

Cliff S Han ${ }^{1}$, Melanie Ann Martin ${ }^{2}$, Armand E K Dichosa ${ }^{1}$, Ashlynn R Daughton ${ }^{3}$, Seth Frietze ${ }^{4}$, Hillard Kaplan ${ }^{5}$, Michael D Gurven ${ }^{6}$, Joe Alcock ${ }^{\text {Corresp. }} 7$

${ }^{1}$ Bioscience Division, Los Alamos National Laborataory, Los Alamos, New Mexico, USA

2 Department of Anthropology, Yale University, New Haven, California, United States

3 Analytics, Intelligence and Technology (A) Division, Los Alamos National Laborataory, Los Alamos, New Mexico, USA

${ }^{4}$ School of Biological Sciences, University of Northern Colorado, Greeley, Colorado, USA

5 Department of Anthropology, University of New Mexico, Albuquerque, New Mexico, USA

${ }^{6}$ Department of Anthropology, University of California, Santa Barbara, Santa Barbara, CA, United States

${ }^{7}$ Department of Emergency Medicine, University of New Mexico, Albuquerque, New Mexico, USA

Corresponding Author: Joe Alcock

Email address: joalcock@salud.unm.edu

Background: Premastication, the transfer of pre-chewed food, is a common infant and young child feeding practice among the Tsimane, forager-horticulturalists living in the Bolivian Amazon. Research conducted primarily with Western populations has shown that infants harbor distinct oral microbiota from their mothers. Premastication, which is less common in these populations, may influence the colonization and maturation of infant oral microbiota, including via transmission of oral pathogens. We collected premasticated food and saliva samples from Tsimane mothers and infants ( 9 to 24 months of age) to test for evidence of bacterial transmission in premasticated foods and overlap in maternal and infant salivary microbiota. We extracted bacterial DNA from two premasticated food samples and 12 matched salivary samples from maternal-infant pairs. DNA sequencing was performed with MiSeq (Illumina). We evaluated maternal and infant microbial composition in terms of relative abundance of specific taxa, alpha and beta diversity, and dissimilarity distances. Results: The bacteria in saliva and premasticated food were mapped to 19 phyla and 400 genera and were dominated by Firmicutes, Proteobacteria, Actinobacteria, and Bacteroidetes. The oral microbial communities of Tsimane mothers and infants who frequently share premasticated food were well-separated in a non-metric multi-dimensional scaling ordination (NMDS) plot. Infant microbiotas clustered together, with weighted Unifrac distances significantly differing between mothers and infants. Infant saliva contained more Firmicutes $(p<0.01)$ and fewer Proteobacteria $(p<0.05)$ than did maternal saliva. Many genera previously associated with dental and periodontal infections, e.g. Neisseria, Gemella, Rothia, Actinomyces, Fusobacterium, and Leptotrichia, were more abundant in mothers than in infants. Conclusions: Salivary microbiota of Tsimane infants 
and young children up to two years of age do not appear closely related to those of their mothers, despite frequent premastication and preliminary evidence that maternal bacteria is transmitted to premasticated foods. Infant physiology and diet may constrain colonization by maternal bacteria, including several oral pathogens. 


\section{Salivary microbiomes of indigenous Tsimane mothers and infants are distinct despite frequent premastication} Cliff S. Han ${ }^{1 *}$, Melanie Martin ${ }^{2 *}$, Armand E. K. Dichosa ${ }^{1}$, Ashlynn Daughton ${ }^{3}$, Seth Frietze ${ }^{4}$, Hillard Kaplan ${ }^{5}$, Michael Gurven ${ }^{6}$, Joe Alcock ${ }^{7}$

1 Los Alamos National Laboratory, Bioscience Division, Los Alamos, NM 87545. Email: han_cliff@lanl.gov

2 Yale University, Dept. of Anthropology, New Haven, CT 06511.

3 Los Alamos National Laboratory, Analytics, Intelligence and Technology Division, Los Alamos, NM 87545.

4 University of Northern Colorado, School of Biological Sciences, Greeley, CO 80639.

5 The University of New Mexico, Dept. of Anthropology, Albuquerque, NM 87131

6 University of California Santa Barbara, Dept. Anthropology, Santa Barbara, CA 93106

7 The University of New Mexico, Dept. of Emergency Medicine, Albuquerque, NM 87131.

* Contributed equally.

Corresponding author: Joe Alcock MD. The University of New Mexico, Dept. of Emergency Medicine, Albuquerque, NM 87131. Phone, 505-272-5062. Email: joalcock@salud.unm.edu

\section{Co-author email addresses:}

Cliff S. Han: han cliff@lanl.gov

Melanie Martin: melanie.martin@yale.edu

Armand E. K. Dichosa: armand@lanl.gov

Ashlynn Daughton: adaughton@lanl.gov

Seth Frietze: frietze@nnmc.edu

Hallard Kaplan: hkaplan@unm.edu

Marchael Gurven: gurven@gmail.com

Joe Alcock: joalcock@salud.unm.edu 
33

34

35

36

37

\section{Abstract}

Background: Premastication, the transfer of pre-chewed food, is a common infant and young child feeding practice among the Tsimane, forager-horticulturalists living in the Bolivian Amazon. Research conducted primarily with Western populations has shown that infants harbor distinct oral microbiota from their mothers. Premastication, which is less common in these populations, may influence the colonization and maturation of infant oral microbiota, including via transmission of oral pathogens. We collected premasticated food and saliva samples from Tsimane mothers and infants ( 9 to 24 months of age) to test for evidence of bacterial transmission in premasticated foods and overlap in maternal and infant salivary microbiota. We extracted bacterial DNA from two premasticated food samples and 12 matched salivary samples from maternal-infant pairs. DNA sequencing was performed with MiSeq (Illumina). We evaluated maternal and infant microbial composition in terms of relative abundance of specific taxa, alpha and beta diversity, and dissimilarity distances.

Results: The bacteria in saliva and premasticated food were mapped to 19 phyla and 400 genera and were dominated by Firmicutes, Proteobacteria, Actinobacteria, and Bacteroidetes. The oral microbial communities of Tsimane mothers and infants who frequently share premasticated food were well-separated in a non-metric multi-dimensional scaling ordination (NMDS) plot. Infant microbiotas clustered together, with weighted Unifrac distances significantly differing between mothers and infants. Infant saliva contained more Firmicutes $(\mathrm{p}<0.01)$ and fewer Proteobacteria $(\mathrm{p}<0.05)$ than did maternal saliva. Many genera previously associated with dental and periodontal infections, e.g. Neisseria, Gemella, Rothia, Actinomyces, Fusobacterium, and Leptotrichia, were more abundant in mothers than in infants.

Conclusions: Salivary microbiota of Tsimane infants and young children up to two years of age do not appear closely related to those of their mothers, despite frequent premastication and preliminary evidence that maternal bacteria is transmitted to premasticated foods. Infant physiology and diet may constrain colonization by maternal bacteria, including several oral pathogens. 


\section{Introduction}

66

67 Premastication is the practice of feeding an infant with food chewed by its mother or other 68 caregivers. Beginning as early as one month of age and continuing for two years or more, the practice has been reported in nearly a third of 119 traditional societies surveyed in ethnographic literature $^{1}$, and is still practiced across diverse societies today (e.g. China ${ }^{1}$, South Africa ${ }^{2}$ ). Premasticating mechanically processes, lubricates, and adds salivary amylase to foods, facilitating digestion and absorption ${ }^{3}$. As such, the practice may have been favored throughout much of human evolution to enhance nutritional availability of weaning foods ${ }^{1}$.

Premastication also has immunological implications. Adult saliva contains lysozyme and antimicrobial peptides that inhibit the growth of foodborne pathogens ${ }^{4}$. Transmission of adult oral microbiota may promote immunotolerance and protect against development of allergic and other non-communicable diseases ${ }^{5}$. Conversely, others have raised concerns that premastication may transfer $\mathrm{HIV}^{2,6}$ and bacteria with cariogenic or pathogenic potential, including group A streptococcus $^{7}$ and Streptococcus mutans ${ }^{8}$.

Vertical transmission of maternal bacteria is a major source of microbial colonization during early life, as demonstrated by the effect of birth mode and breastfeeding on developing infant gut microbiomes $^{9}$. The extent to which infant oral microbial communities are varyingly influenced by maternal and other environmental exposures, however, is less clear. For example, maternal oral community structure at birth does not appear to influence initial colonization of infant oral microbiota ${ }^{10}$ and mothers and infants may harbor distinct oral microbial communities for at least the first year of life ${ }^{11,12}$. These differences suggest strong developmental constraints on infant oral microbiota, as only with increasing age and dental eruption do their microbial communities become more complex and show greater overlap with those of their mothers ${ }^{13}{ }^{14}$. At the same time, maternal transmission of $S$. mutans has been detected in pre-dentulous infants, with colonization mediated by frequency of maternal-infant contact and maternal levels of the bacteria $^{14-16}$. 
95 Differences in maternal-infant behaviors may therefore variably influence rates of maternal 96 bacterial transmission across and within populations. Caregiver behaviors that can transmit 97 salivary bacteria to infants include kissing, sharing food and utensils, and even "licking clean" 98 infants' pacifiers ${ }^{5}$. We propose that premastication may transmit a relatively greater abundance 99 and/or diversity of maternal bacteria relative to these other behaviors, however, due to the 100 amount of saliva transferred to chewed food and the number of oral features potentially sampled 101 during chewing.

103 The Tsimane are an indigenous population of forager-horticulturalists living in the Bolivian 104 Amazon. Tsimane patterns of pathogen exposure, hygiene, diet, and reproduction contrast 105 sharply with those observed in more Westernized populations, and are accordingly expected to influence patterns of microbial acquisition and composition. Most Tsimane lack access to clean water, electricity, plumbing, or quality medical care, and gastrointestinal and respiratory diseases are endemic ${ }^{17,18}$. Oral health and hygiene practices are generally poor, with the result that children frequently exhibit caries in their deciduous teeth, and adults exhibit numerous missing teeth.

112 The Tsimane diet consists primarily of cultivated carbohydrates supplemented with foraged 113 game and fish, with relatively few processed foods ${ }^{19}$. Almost universally, Tsimane infants are

114 delivered vaginally at home and breastfed on demand throughout the day and night. On average 115 they are exclusively breastfed for about 4 months and weaned at about 19 months ${ }^{20}$. Mothers are 116 the primary caregivers responsible for feeding children up through the first two years of life.

117 Tsimane mothers do not provision infants with commercially prepared foods or cereals. In 118 general, infants sit with their mothers during meals, and are spoon- or hand-fed small portions of 119 whatever their mothers are eating. Before six months of age the infant diet may be primarily 120 restricted to liquids and broths, with liquids sometime given mouth-to-mouth. Premastication is 121 observed up through the first two years of life, and is primarily restricted to foods deemed too 122 hot, too dry, or to pose choking hazards (e.g. stews, roasted plantains, meat, and fish) 123

124 In a recent survey on the feeding practices of 132 Tsimane infants and young children aged 0-35 125 months (from here on "infants"), $84 \%$ of mothers reported premasticating foods for their infants 
126 at least once, and 54\% reported premasticating foods the day prior ${ }^{21}$ Fathers and siblings were

127 observed sharing snacks with infants on occasion, but were not observed or systematically

128 documented to premasticate.

129

130 As preliminary evidence that premastication may influence oral bacteria transmission and

131 microbial relatedness among mothers and infants, we studied microbiota extracted from

132 premasticated food and matched maternal and infant saliva samples of Tsimane subjects. . We

133 hypothesized that owing to the high frequency of premastication, salivary microbiomes of related

134 Tsimane mother-infant pairs would be more similar to one another than those of unrelated pairs.

135 Findings from this study may advance understanding of infant oral microbial development in

136 non-Western contexts. Results may also have direct implications for Tsimane infant health, as on

137 the one hand premastication may buffer against nutritional losses and pathogen exposure

138 associated with weaning transitions, but on the other may increase already high risks of oral

139 pathogen transmission and associated dental diseases.

\section{Methods}

\section{Study design}

144 Maternal and infant saliva samples were collected from 12 dyads in three villages. The

145 participating dyads were part of an ongoing research project being conducted by Author 2 on

146 Tsimane infant feeding transitions and health outcomes. All infants were reported by their

147 mothers to still receive premasticated foods. For 10 of 12 infants, premastication in the two

148 weeks prior to sample collection was also documented in routinely collected 24-hour dietary

149 recalls (Table 1). . All infants were currently breastfeeding, though measures of daily breast

150 milk intake were not collected or estimated. Neither maternal nor infant subjects had taken

151 antibiotics in the week prior to sample collection. Subjects were selected as a matter of

152 convenience, as in their respective communities they lived in close proximity to the researcher's

153 field base.

154

155 Mothers and infants varied in their respective ages and dental health (Table 1). The following 156 maternal subjects were sisters: Mother.1/Mother.3; Mother.10/Mother.11; Mother.13/Mother.15. 
Table 1. Metadata of Mothers and Infants.

\begin{tabular}{|c|c|c|c|c|c|c|c|c|}
\hline Subject & Sample & Sex & Village & Age (years) & \# Teeth & \# Caries & Sampling method & Most recent premasticated foods documented \\
\hline Infant.1 & BSA1 & $\mathrm{F}$ & 1 & 1.3 & 8 & 0 & Buccal swab & 1 day prior: fish, meat stew, plantain \\
\hline Infant.2 & BSA2 & $\mathrm{F}$ & 1 & 1.1 & 6 & 0 & Buccal swab & 1 day prior: rice, plantain, meat stew \\
\hline Infant.3 & BSA3 & $\mathrm{F}$ & 1 & 0.8 & 4 & 0 & Buccal swab & 1 day prior: plantain, meat \\
\hline Infant.4 & BSA4 & $\mathrm{M}$ & 1 & 1.5 & 14 & 0 & Buccal swab & 1 day prior: fish stew \\
\hline Infant.8 & BSA8 & $\mathrm{M}$ & 2 & 2.0 & 16 & 1 & Buccal swab & 1 day prior: fish \\
\hline Infant.9 & BSA9 & $\mathrm{F}$ & 2 & 1.7 & 15 & 8 & Buccal swab & 1 day prior: fish \\
\hline Infant.10 & BSA10 & $\mathrm{M}$ & 2 & 1.7 & 16 & 0 & Buccal swab & N/A \\
\hline Infant.11 & BSA11 & $\mathrm{M}$ & 2 & 1.1 & 8 & 0 & Buccal swab & N/A \\
\hline Infant.12 & BSA12 & $\mathrm{M}$ & 3 & 0.8 & 1 & 0 & Buccal swab & 11 days prior: rice, plantain \\
\hline Infant.13 & BSA13 & $\mathrm{M}$ & 3 & 1.2 & 10 & 0 & Buccal swab & 4 days prior: rice \\
\hline Infant.14 & BSA14 & $\mathrm{M}$ & 3 & 1.1 & 9 & 0 & Buccal swab & 10 days prior: plantain, meat, fish stew \\
\hline Infant.15 & BSA15 & $\mathrm{M}$ & 3 & 0.9 & 5 & 0 & Buccal swab & 10 days prior: stew, plantain, meat \\
\hline Mother.1 & MSA1 & $\mathrm{F}$ & 1 & 20.1 & 22 & 1 & Buccal swab & \\
\hline Mother.2 & MSA2 & $\mathrm{F}$ & 1 & 18.8 & 24 & 0 & Buccal swab & \\
\hline Mother.3 & MSA3 & $\mathrm{F}$ & 1 & 36.3 & 18 & 0 & Buccal swab & \\
\hline Mother.4 & MSA4 & $\mathrm{F}$ & 1 & 21.3 & 27 & 1 & Buccal swab & \\
\hline Mother.8 & MSA8 & $\mathrm{F}$ & 2 & 29.6 & 22 & 1 & Buccal swab & \\
\hline Mother.9 & MSA9 & $\mathrm{F}$ & 2 & 22.7 & 30 & 0 & Buccal swab & \\
\hline Mother.10 & MSA10 & $\mathrm{F}$ & 2 & 24.6 & 24 & 1 & Buccal swab & \\
\hline Mother.11 & MSA11 & $\mathrm{F}$ & 2 & 26.8 & 19 & 1 & Buccal swab & \\
\hline Mother.12 & MSA12, FD12 & $\mathrm{F}$ & 3 & 24.9 & 32 & 1 & Expectoration & \\
\hline Mother.13 & MSA13 & $\mathrm{F}$ & 3 & 27.7 & 15 & 8 & Expectoration & \\
\hline Mother.14 & MSA14, FD14 & $\mathrm{F}$ & 3 & 31.6 & 25 & 1 & Expectoration & \\
\hline Mother.15 & MSA15 & $\mathrm{F}$ & 3 & 25.2 & 21 & 1 & Expectoration & \\
\hline
\end{tabular}

Subjects from the same family have the same indexing number. BSA, infant saliva; MSA, mother saliva; FD, premasticated food.

159 'Most recent premasticated foods' were documented from subjects' most recent 24-hour dietary recalls. Dietary recall was not

160 collected for subjects BSA10 and BSA11, though at sample collection their mothers reported still premasticating all types of foods.

161 Premasticated food sample FD14 was collected approximately 40 minutes after cooking, and was chewed for 17 seconds. Sample

162 FD12 was collected 50 minutes after cooking, and was chewed for 8 seconds. 
165

166 The four mothers from Village 3 who participated in the controlled experiment to collect

167

168

169

170

171

172

173

174

175

176

177

178

179

180

181

182

183

184

185

186

187

188

189

190

191

192

193

194

195

premasticated food samples were also neighbors and frequently exchanged food or shared meals.

As no dental records were available, we report the total number of teeth per subject rather than

missing teeth. We assume that incomplete tooth counts for adult subjects (less than 32 teeth)

primarily represent missing teeth due to dental disease and/or manual extraction, whereas incomplete tooth counts in infants (less than 20) primarily reflect different stages of tooth eruption.

\section{Sample collection}

Saliva. Twenty-four saliva samples were collected from 12 Tsimane mother-infant pairs from three different villages (Table 1). Saliva samples from subjects in illages 1 and 2 were collected by placing a sterile buccal cell collection swab (Catch-All ${ }^{\mathrm{TM}}$ Sample Collection Swab, Epicentre $\left.{ }^{\circledR}\right)$ on the subject's tongue for five seconds. The swabs were kept in the sterile collection tubes at ambient temperature and transported back to a field laboratory. The buccal swabs were transferred to cryotubes and stored in liquid nitrogen within approximately 30 minutes of sample collection.

Saliva and premasticated food samples were collected from participants in Village 3 only, with saliva samples collected prior to food consumption. who. Infant saliva samples in this group were collected using the buccal cell collection method described above. The maternal saliva sampleswere collected via expectoration rather than buccal swab in order to duplicate the sampling method used to collect premasticated food. Mothers were asked to expectorate into disposable plastic cups, and the researcher transferred the saliva on site into individually prelabeled $2 \mathrm{ml}$ cryotubes using disposable plastic pipettes.

Premasticated food. A traditional Tsimane stew ( "jo'na”, made from green plantains and meat) was prepared by one of the participants in her home, with ingredients and cookware supplied by Author 2 and the participant. All cookware and utensils were cleaned prior to use with water and a $70 \%$ alcohol solution. The stew was made by boiling approximately $1 \mathrm{~kg}$ of dried beef, 20 
196 unripe plantains, and vegetable oil and salt to taste. Cooking water was sourced from a local

197 river, and the stew was boiled for approximately 19 minutes over an open flame.

198

199 Five control samples of unchewed food were taken from the pot approximately 17 minutes after

200 the pot was removed from heat. The attending author served food to and gathered samples from

201 each of the four participants. Mothers were instructed to premasticate food just as they normally

202 would for their infants, and then expectorate the premasticated bite into a disposable plastic cup.

203 The unchewed and premasticated food samples were transferred into pre-labeled $2 \mathrm{ml}$ cryotubes

204 using wooden oral tongue depressors. The samples were kept in an insulated bag at ambient

205 temperature during the sampling collection process, and then returned to the field site laboratory

206 for immediate storage in liquid nitrogen. The time between sample collection and storage for all

207 saliva and food samples from Village 3 ranged from approximately $1-1.75$ hours. All samples

208 were transported on dry ice to the U.S. and stored at $-80{ }^{\circ} \mathrm{C}$ until analysis.

209

210

\section{DNA isolation}

212 Following manufacturers' protocols, a PSP Saliva Gene Kit (STRATEC Molecular GmbH,

213 Germany) was used to extract DNA from the saliva samples with added lysozyme for better lysis

214 of Gram positive bacterial cells as suggested by the supplier. And a PowerLyzer ${ }^{\circledR}$ UltraClean ${ }^{\circledR}$

215 Microbial DNA Isolation Kit (MoBio Cat\# 12255-50, MO BIO Laboratories, Carlsbad, CA) was

216 used to extract DNA from the food samples. DNA concentrations were measured with a Qubit

217 Fluorometer (Life Technologies, Grand Island, NY). Only two of the five control food samples

218 were selected for DNA analysis, and did not yield sufficient DNA for PCR amplification. Two of

219 the four premasticated food samples (corresponding to Mother.13 and Mother.14, Table 1) also

220 failed to yield sufficient DNA for further analysis. PCR amplification and sequencing was

221 therefore limited to the 24 saliva samples and only two premasticated food samples (FD12 and

222 FD14, Table 1).

223

224 PCR amplification and sequencing

225 The V4 region of the bacterial 16S rRNA gene was amplified with a NEXTflex 16S rRNA gene

226 V4 Amplicon-Seq Kit (with 48 barcodes) (BIOO Scientific, Austin, TX) following the 
227 manufacturer's instructions. The kit produces amplicons of 250 bases that can be sequenced with

228 Illumina technology from both end with significant overlap. The PCR primers are V4_515f

229 adaptor 5'AATGATACGGCGACCACCGAGATCTACACTATGGTAATTGT

230 GTGCCAGCMGCCGCGGTAA3' and V4_806r adaptor

231 5'CAAGCAGAAGACGGCATACGAGAT XXXXXXXXXXXX AGTCAGTCAGCC

232 GGACTACHVGGGTWTCTAA3', with "X" indicating indexing sequencing capable to code up

233 to 1,152 individual samples. Sequences at 5' ends of the primers are adaptors for Illumina

234 sequencing, those at $3^{\prime}$ ends are $16 \mathrm{~S}$ rRNA gene primers. In total, 50 ng DNA was used as a

235 template for each reaction. The amplified DNAs were verified on a gel and pooled at an equal

236 molar ratio. The pooled DNA samples were purified with Agencourt AMPure beads. Sequencing

237 was performed on a MiSeq (Illumina, San Diego, CA).

238

239 16S rRNA gene sequence data processing

240 An analysis of the 16S rRNA gene sequencing data was performed using the mothur software

241 package $^{22}$. Mothur was selected for ease of installation, clear and brief instructions, and

242 compatibility with other software ${ }^{23}$. Initially, the paired end sequences were assembled together,

243 and any sequences with ambiguous bases (32\% of them) were removed. To ease the sequence

244 classification process, unique sequences were collected. The collection was further reduced by

245 choosing representatives of near-identical sequences with fewer than 2 different bases. Chimeric

246 sequences $(\sim 18 \%)$ were removed with $\mathrm{UCHIME}^{24}$. Sequences left were then aligned using the

247 SILVA databases supplied in mothur, and those sequences classified as Chloroplast,

248 Mitochondria, Archaea, Eukaryota, or Unknown were removed (293 of them). The sequence

249 yield and indexing codes for all samples analyzed are given in Supplementary Table 1.

250

251 The unique sequence collection was clustered into operational taxonomic units (OTUs), with a

252 similarity of $97 \%$. OTU with single or double sequences were removed. Each OTU was assigned

253 a taxonomic classification at all levels, from phylum to genus, using the reference Ribosomal

254 Database Project (RDP) database provided in mothur. Alpha diversity was analyzed with

255 Rarefraction curve deduced with tools from mothur. The count table was normalized with

256 cumulative count scaling implemented in metagenomeSeq ${ }^{25}$. Distance ordination was done with

257 normalized data. Total-sum scaling was followed for taxa comparison. The dissimilarity 
258 distances between samples were calculated in mothur using the dist.shared command. Thetayc

259 distance matrices were used to compare phylogenetic distances among infants and mothers, and

260 to examine differences in phylogenetic distance within and between dyads ${ }^{26}$. Weighted Unifrac

261 distance metrics ${ }^{27}$ were calculated to compare the microbial communities of mothers and infants,

262 which result is also confirmed with ANOSIM $^{28}$. Distance ordination was ploted with non-metric

263 multidimensional scaling method that implemented in metagenomeSeq ${ }^{25}$. Finally, we attempted

264 to identify some OTUs of interest at the species level using the HOMD library with the online

265 16S rRNA gene sequence identification service ${ }^{29}$.

266

267 Statistical analyses

268 Student t-tests were used to compare alpha diversity, abundances at different phylogenetic levels, 269 and phylogenetic distances between mothers and infants. The Shapiro-Wilk test was used to

270 test the normality of the distribution of taxonomic data (the abundance of most common

271 taxa). Of 91 data sets (12 samples each, infant and mother considered separately) 57\%

272 were normally distributed $(\mathbf{p}<\mathbf{0 . 0 5})$. Mann-Whitney $U$ tests were used for data sets that

273 were not normally distributed. P-values were further adjusted for multiple comparisons using

274 the false discovery rate method ${ }^{30}$. All analyses were performed in R. Food samples were not

275 statistically evaluated since only two samples were sequenced.

276

277 Data availability

278 Sequence data was submitted to Sequence Read Archive at National Center for Biotechnology

279 Information and available under Bioproject Accession PRJNA320081 with Group Accession

280 Number SRP074186.

281

282 Ethics approval and consent

283 This study was approved by the Human Subjects Committee of the University of California

284 Santa Barbara (ANTH-GU-MI-010-3U) and the Human Subject Research Review Board of Los

285 Alamos National Laboratory (LANL-14-11 X). The Tsimane Health and Life History Project

286 maintains annual agreements with the local municipal government of San Borja, the Hospital of

287 San Borja, and the Tsimane governing council, El Gran Consejo Tsimane', to conduct research 
288 with the Tsimane. Maternal subjects gave verbal informed consent for themselves and their

289 infants to participate prior to sample collection.

290

291 Results

292

293 General characteristics of Tsimane mothers' and infants' oral microbiomes

294

295 The bacteria in the saliva and premasticated food samples belonged to 18 different phyla and 341

296 genera (Supplementary Table 2). The main bacterial phyla were Firmicutes (with the

297 predominant genus Streptococcus), Proteobacteria (Neisseria), Actinobacteria (Actinomyces),

298 and Bacteroidetes (Prevotella). Other phyla included Fusobacteria, Spirochaetes, TM7,

299 Tenericutes, SR1, and Verrucomicrobia. The relative abundance of each phylum was different

300 between the saliva and food samples of mothers and infants (Figures $1 \&$ 2). Infant saliva

301 samples contained more Firmicutes $(\mathrm{p}<0.01)$ and less Fusobacteria and Spirochaetes $(\mathrm{p}<0.05)$

302 than did maternal samples. The mean relative abundance of the most abundant families across all

303 saliva samples are listed in Supplementary Table 3.

304

305 Thirteen genera—Streptococcus, Neisseria, unclassified Pasteurellaceae, Gemella, Prevotella

306 Leptotrichia, Actinomyces, Rothia, Veillonella, Sphingomonas, and unclassified genera from the

307 Leptotrichiaceae, Flavobacteriaceae, and Lactobacillales families - were observed in all saliva

308 samples. The most common genus, Streptococcus, has 4 OTUs (OTU0001, OTU0003, OTU0029

309 and OTU0084) and was dominant in 92\% (22/24) of saliva samples. OTU0001 is identical to

310 Streptococcus mitis, S. oralis, S. infantis, and S. mitis bv. 2 based on comparison with the Human

311 Oral Microbiome Database (HOMD) ${ }^{29}$. We could not distinguish among these species, as the V4

312 regions of their 16S rRNA gene sequences are identical. It is possible that samples harbored

313 more than one of these species. The average abundance of OTU0001 was $41.3 \%$ in infants'

314 saliva and $25.2 \%$ in mothers' saliva (adjusted $\mathrm{p}<0.01$ ). OTU0003 is identical to $16 \mathrm{~S}$ rRNA gene

315 sequences of S. salivarius and S. vestibularis in HOMD. This OTU was about a third as abundant

316 as OTU0001, and twice as abundant in infants $(22.1 \%)$ as in mothers $(11.5 \%)(\mathrm{p}<0.01)$.

317 OTU0029 is identical to $S$. Intermedius. This OTU was more abundant in mothers' saliva $(0.8 \%$

318 vs $0.1 \%$ in infants), but the difference is not statistically significant. OTU0084 matches 
319 Streptococcus sp. oral taxon 487 in HOMD at 98.8\% and was found in only two infant and four

320 maternal samples. Overall, infants' saliva contained significantly more Streptococcus $(65.0 \%$ vs

$32136.9 \%, \mathrm{p}<0.01)$ and less Leptotrichia (0.2\% vs 2.4\%, $<<0.01)$ and Catonella (2 infants and 11

322 mothers have it, $\mathrm{p}<0.01$ ) than mothers' saliva after adjusting for false discovery rate.

323

324 Microbial populations of premasticated foods in relation to donor salivary populations

325 Bacterial populations from the two premasticated food samples that could be sequenced were 326 more similar to those of their donors than to those of other mothers, although this finding only 327 trends toward statistical significance $(\mathrm{p}=0.065)$, likely due to the small sample size. Differences

328 in dissimilarity distances of the foods samples may also reflect individual differences in chewing

329 "style" and duration. The sample that was chewed for 17 seconds had a smaller dissimilarity

330 distance than that of the sample chewed for 8 seconds ( 0.061 vs 0.191$)$, suggesting more donor

331 bacteria may be incorporated in food with increased chewing time.

332

333

Despite within-subject similarities between premasticated food and donor saliva, 16S rRNA gene

334 sequencing revealed distinct differences between chewed food and saliva samples

335 (Supplementary Table 4), which were both collected via expectoration. Sixty OTUs were

336 enriched more than 5-fold in premasticated food compared with saliva samples, and 36 OTUs

337 were only observed at $>0.01 \%$ in premasticated food samples. Some bacteria in the food samples

338 may have been incorporated through the surface water used in food preparation, as we identified

339 bacteria associated with plants, soil, and water reservoirs in the premasticated food samples.

340 However, we did not yield sufficient DNA from the original unchewed food for 16S rRNA gene

341 sequencing in two attempts, suggesting cooking decreased the availability of bacteria or bacterial

342 DNA in the food samples.

343

344 Relatedness of maternal and infant salivary microbiomes

345 We assessed relatedness of maternal and infant salivary microbiota by comparing alpha

346 (intrasample) diversity and beta (intersample) diversity among saliva samples.

347 The internal sample alpha diversity was measured by rarefaction analysis (Supplementary

348 Figure 1). On average, infant saliva samples had 41 OTUs when sampling 3148 sequences, 349 whereas maternal samples had $87(\mathrm{p}<0.01)$. The lower diversity of infants' saliva paralleled 
350 greater dominance of Streptococcus. Intra-sample diversity was not correlated with age or

351 number of teeth when analyzing mothers and infants separately, but was correlated with age

352 when analyzing the two groups together $(r=0.75, \mathrm{p}<0.01)$. Alpha diversity differed among

353 maternal subjects according to sample collection method:113 \pm 24 for expectorated samples and

$35475 \pm 22$ for samples collected via buccal swab $(\mathrm{p}=0.01)$.

355

356 Both ANOSIM and weighted Unifrac analysis indicated that mothers and infants had different 357 salivary microbial community structures $(\mathrm{p}<0.01)$. However, the difference was not significant

358 when calculated with unweighted Unifrac analysis, which indicated that the abundance of shared

359 OTUs contributed more to the weighted Unifrac calculation. The average distances among infant

360 saliva samples $(0.30 \pm 0.18)$ were significantly shorter than those among maternal samples $(0.48$

$361 \pm 0.21)(\mathrm{p}<0.01)$, indicating greater similarity among infant microbial communities than among

362 maternal communities (Figure 4). Average distances calculated for related mother-infant pairs

363 (0.45) were not significantly shorter than those of unrelated mother-infant pairs (0.47),

364 suggesting community structures of related pairs were no more similar than those of unrelated

365 pairs (Figure 4). A non-metric multi-dimensional scaling ordination (NMDS) plot made using a

366 Thetayc tree further shows that infants' salivary microbiomes were more similar in community

367 structure to one another than to maternal microbiomes (Figure 3).

368

369 We also calculated the average distance between each infant with all possible combinations of

370 mothers. Across infants, this average distance was negatively correlated with infant age $(r=-0.64$,

$371 \mathrm{p}<0.05$, Supplementary Figure 3A). The average distance between each maternal sample and

372 all infant samples, however, was not associated with maternal age $(r=-0.33, p=0.29)$

373 (Supplementary Figure 3B). Tsimane salivary microbial communities may therefore become

374 more adult-like during late infancy (9-24 months), but there is little age-associated variation in

375 community structure during adulthood (18-41 years) in this study group.

376

377 Oral pathogens identified in maternal and infant saliva samples

378 Chronic dental infections are common in Tsimane people, as evidenced by numerous caries and

379 tooth loss in our maternal subjects (Table 1). We searched for pathogens associated with dental

380 disease in maternal and infant samples, which could indicate vertical transmission. Using the 
381 HOMD library, OTU0120 matched S. mutans at 100\%; OTU0143, T. denticola at 98\%;

382 OTU0068, T. forsythia at 97.2\%; and OTU0033, P. gingivalis at 99.2\%. Because OTU0068 and

383 OTU0143 matched target species at a relatively low level, we restricted our analyses to

384 OTU0033 (P. gingivalis) and OTU0120 (S. mutans) and traced them across mother-infant dyads.

385 However, these species-level identifications are limited because we did not perform confirmatory

386 tests to identify these OTUs as pathogens.

387

388 In normalized results, OTU0033 ( $P$. gingivalis) was observed in 11 mothers' saliva samples 389 (92\%, MSA1, MSA8, MSA12, MSA13, MSA15), but not in any of the infants' samples 390 ( $\mathrm{p}<0.01)$. OTU0120 (S. mutans) was observed in 7 infants' saliva samples (BSA1, BSA11, 391 BSA12, BSA13, BSA14, BSA15, BSA8) and 8 mothers' samples (MSA1, MSA10, MSA11, 392 MSA12, MSA13, MSA14, MSA15, MSA9). The frequency of $S$. mutans did not significantly 393 differ across maternal and infant samples $(\mathrm{p}=0.24)$. Three pairs of mother-infant samples $(\mathrm{BSA} 8$ 394 and MSA8, BSA9 and MSA9, BSA10 and MSA10) did not match each other in S. mutans status, 395 and the remaining pairs (9) did. The occurrence and abundance of the two possible pathogens 396 were not correlated with age, the number of teeth, dental caries, or the loss of teeth within each 397 group. There were 754 S. mutans reads from infant saliva sample BSA14, compared with fewer 398 than 5 in other samples, but the subject providing this sample had no observable dental caries at 399 the time of sampling.

400

401 Fourteen of 22 genera associated with gingivitis were identified in $50 \%$ or more of maternal 402 samples (Supplementary Table 5). Seven of these 14 genera were identified in only 1/3 or less of 403 infant samples, and no Eubacterium or Johnsonella were identified in any infant sample 404 (Supplementary Table 5). Fusobacterium, Leptotrichia, Porphyromonas, and Prevotella were 405 identified in at least $83 \%$ of both maternal and infant samples (Supplementary Table 5). All were 406 more abundant in the maternal samples (Supplementary Figure 2). With the exception of 407 Tannerella, the gingivitis-associated OTUs positively identified in infant samples were also 408 identified in the corresponding maternal samples, but not vice versa.

409

\section{Discussion}


411 Maternal-offspring transmission of salivary bacteria through premastication may have been

412 universal in ancestral human populations and remains common today ${ }^{1}$. This is the first study to

413 document the microbiome of premasticated foods and to examine relationships between maternal

414 and infant salivary microbiota in an indigenous population with frequent sharing of pre-chewed

415 food.

416

417 Overall, the patterns of oral microbial composition observed for the Tsimane support existing

418 findings for mothers and infants in diverse populations. The 5 most predominant phyla observed

419 for Tsimane subjects (Figure 1) were the same as those previously observed in a sample of U.S.

420 children aged 3-18 13 and in a sample of U.S. mothers and their infants aged 3-6 months old ${ }^{11}$,

421 Across all three study populations, Firmicutes was the most abundant phylum, with

422 Streptococcus predominating samples and found at higher relative abundance in infants and

423 children than in adult subjects. The relative abundance of Bacteroidetes in samples from Tsimane

424 children aged 9-24 months was lower as compared to that of older U.S. children but higher

425 compared to that of younger U.S. infants, which may support the observation that this phylum

426 increases with age ${ }^{11}$. Dissimilarity distances across Tsimane infant and maternal samples also

427 decreased with age (Supplementary Figure 3), consistent with previous observations that oral

428 microbial composition changes quickly during childhood ${ }^{31}$, likely due to changes in dentition

429 and diet. The most predominant 13 genera observed among Tsimane samples are also consistent

430 with the previous observation of the existence of a core oral microbiome ${ }^{32,33}$.

431

432 The bacteria extracted from the premasticated food samples in our study appear to have been at

433 least partially derived or modified by chewing. Mastication has been shown to release

434 Acinetobacter ${ }^{34}$ and Staphylococcus ${ }^{35}$ from the subgingival pocket. The chewed food samples in

435 this study harbored a greater diversity of bacteria than corresponding subject saliva samples,

436 which may reflect contact with dental, lingual, buccal, and subgingival surfaces during chewing.

437 This finding tentatively supports our proposal that premastication may transmit a greater

438 diversity of oral bacteria - including potential pathogens - than more superficial modes of

439 salivary exchange, including non-romantic kissing and utensil-sharing. However, our inferences

440 are confounded by the fact that DNA from saliva and premasticated food were isolated using

441 different kits $^{33}$, which were selected to optimize the bacterial DNA yield respective to the sample 
442 sources. This limitation can be remedied by using consistent collection and laboratory protocols

443 in future studies.

444

445 Despite evidence that maternal bacteria is incorporated into premasticated food, salivary 446 microbiomes of related mother-infant pairs known to share premasticated foods were no more 447 similar than those of unrelated pairs. These results are in line with existing cross-cultural 448 research showing distinct clustering between mothers and infants with respect to both gut ${ }^{36-39}$ 449 and oral microbiomes ${ }^{10,11}$. Evidence of transmission of oral pathogens between Tsimane mothers 450 and infants was also weak. The abundance of Actinomyces, Leptotrichia, and Fusobacterium451 previously associated with dental, periodontal, and other infections $40-42$ — was significantly lower 452 for infants as compared to their mothers. Bacteria identified as S. mutans occurred in two infants 453 of mothers who harbored the bacteria, but also in three infants of mothers who did not.

454

455

456

457

458

459

460

461

462

463

464

465

466

467

468

469

470

471

472
Ultimately, several factors may limit the influence of premastication on Tsimane infant salivary microbiomes, as well as our ability to detect these influences in our study. First, we do not have precise data on the frequency of premastication prior to sampling or over time in our subjects. However, even frequent maternal salivary transfer via premastication may have only transitory effects on infant microbial communities, as is suggested by a recent study of bacterial transmission following intimate kissing in adults ${ }^{43}$. Second, while infants are continually exposed to novel environmental bacteria, acquisition of particular species and overall community structure in early life appears tightly constrained by developing features of the oral cavity ${ }^{11,44,45}$. Infants in our sample exhibited various stages of dental eruption, which may have limited opportunities for colonization by maternal bacteria. Acquisition of $S$. mutans, for example, may occur during a well-delineated "window of infectivity" corresponding with tooth eruption" though S. mutans has been detected in predentulous infants, and at higher proportions among infants of infected vs. non-infected caregivers ${ }^{46}$.

Finally, intensive breastfeeding in this population may be a significant factor shaping infant oral microbial composition, oral pathogen transmission, and disease risk. Breast milk contains maternally-derived bacteria and nutritional and antimicrobial factors that may uniquely influence 
473 or interact with oral bacteria to shape oral community composition in breastfed infants ${ }^{47,48}$. For

474 example, certain isolates of $S$. oralis, $S$. sanguis, and $S$. mitis have been shown to produce IgA1

475 protease in neonates, which given abundant IgA in breast milk, may confer an ecological

476 advantage over other bacteria that lack this enzyme ${ }^{49}$. In vitro studies have also demonstrated

477 that breastmilk mixed with neonatal saliva stimulates hydrogen peroxide to selectively inhibit the

478 growth of $S$. aureus and Salmonella $\mathrm{spp}^{50}$.

479

480 Though some research suggests breastfeeding increases risks of early childhood caries-

481 particularly when breastfeeding is prolonged after one year of age and includes night nursing ${ }^{51}$

482 evidence for this association is inconsistent and frequently confounded by undocumented

483 variability in infant diets, oral hygiene, and maternal oral health ${ }^{52,53}$. It is of particular interest

484 then that in this study caries were observed in only two infant subjects, though all (at up to two

485 years of age) were still breastfeeding, co-sleeping with their mothers. That is, we have no

486 evidence that these behaviors increase disease risk in the context of Tsimane nutritional and

487 disease ecologies. Similarly, caries can occur in the absence of specific associated pathogens

488 such as $S$. mutans ${ }^{54}$, and ultimately many bacterial species, or the balance of particular species,

489 play a role in the etiology of caries ${ }^{55-57}$. Frequent of disease-associated bacteria through

490 premastication, therefore, may not be sufficient to induce disease, as such bacteria may be

491 "pathogenic" only in the context of the habitat offered by their host.

492

493 The Tsimane do have generally poor oral health, as is evident from the number of caries and

494 missing teeth observed in maternal subjects, and which we have also observed in older children

495 and adolescents. With increasing age and dental maturation, Tsimane infant oral microbiomes

496 become more adult like, while the frequency of breastfeeding and premastication gradually

497 decline (Martin 2015, Veile et al., 2014). Younger children, therefore, may be protected by

498 prebiotic and immunological constituents in breast milk and maternal saliva that selectively

499 promote the growth of some commensals while inhibiting establishment of pathogens.

500 Additional research is needed, however, to identify population-specific risks of oral pathogen

501 transmission and related disease etiologies, as well as to draw firm conclusions about the relative

502 costs of disease exposure vs. potential nutritional or immunological benefits from

503 premastication ${ }^{48}$. Comparative studies, larger sample sizes, and more precise measures of 
504 premastication frequency, breastfeeding intensity, and dietary composition are also necessary to

505 better understand how these behaviors intersect in shaping infant oral microbial development and 506 disease risk at different stages of dentition.

507

508

509 Conclusion

510 Oral microbiomes of Tsimane infants and young children up to two years of age do not appear

511 closely related to those of their mothers, despite frequent premastication and evidence that

512 maternal oral bacteria is transmitted to premasticated foods. Tsimane mothers displayed poor

513 oral health and abundant oral pathogens, but these bacteria were found less frequently in infant

514 samples and did not systematically co-occur within dyads. Our conclusions are limited by a

515 small sample size and two methodological inconsistencies that may have influenced results:

516 differing methods of salivary sample collection for maternal subjects, and the use of different

517 DNA kits for salivary and food samples. Future research should avoid these limitations,

518 simultaneously examine oral microbial composition in a comparative sample of infants who do

519 not receive premasticated foods, and consider the confounding influence of factors such as the

520 stage of dental eruption and intensity and duration of breastfeeding.

521

522 Despite these limitations, the patterns of salivary microbial composition observed for Tsimane

523 mothers and infants - in terms of most abundant phyla and pattern of maternal and infant

524 clustering - are broadly consistent with those previously observed for mothers and young infants

525 in the U.S ${ }^{11}$. However, we did observe differences in the relative abundance of distinct phyla and

526 genera across these two sample populations, which may reflect differences in pathogen exposure,

527 diet, and oral hygiene. The current study thus highlights the need for broad sampling in human

528 microbiome research. Cross-cultural studies of populations with diverse diets, disease ecologies,

529 behaviors, and socioeconomic conditions are especially informative in improving our

530 understanding of how human oral microbiomes are acquired and shaped across the lifespan.

531

532

533

534

535

536
Acknowledgements: We thank David Sela and anonymous reviewers for their helpful critiques on earlier drafts of the manuscript. We are grateful for the support of Tsimane participants and the staff of the Tsimane Health and Life History Project in Bolivia. 
537

538

539

540

541

542

543

544

545

546

547

548

549

550

551

552

553

554

555

556

557

558

559

560

561

562

563

564

565

566

567

568

569

570

571

572

573

574

575

576

577

578

579

580

581

Supplementary information is available at $\mathrm{xxx}$.

\section{References}

1. Pelto, G.H., Zhang, Y. \& Habicht, J.P. Premastication: the second arm of infant and young child feeding for health and survival? Matern Child Nutr 6, 4-18 (2010).

2. Maritz, E.R., Kidd, M. \& Cotton, M.F. Premasticating food for weaning African infants: a possible vehicle for transmission of HIV. Pediatrics 128, e579-590 (2011).

3. Humphrey, S.P. \& Williamson, R.T. A review of saliva: normal composition, flow, and function. J Prosthet Dent 85, 162-169 (2001).

4. Ellison, R.T., 3rd \& Giehl, T.J. Killing of gram-negative bacteria by lactoferrin and lysozyme. The Journal of clinical investigation 88, 1080-1091 (1991).

5. Hesselmar, B., Sjoberg, F., Saalman, R., Aberg, N., Adlerberth, I. \& Wold, A.E. Pacifier Cleaning Practices and Risk of Allergy Development. Pediatrics (2013).

6. Gaur, A.H., Dominguez, K.L., Kalish, M.L., Rivera-Hernandez, D., Donohoe, M., Brooks, J.T. \& Mitchell, C.D. Practice of feeding premasticated food to infants: a potential risk factor for HIV transmission. Pediatrics 124, 658-666 (2009).

7. Steinkuller, J.S., Chan, K. \& Rinehouse, S.E. Prechewing of food by adults and streptococcal pharyngitis in infants. J Pediatr 120, 563-564 (1992).

8. Berkowitz, R.J., Turner, J. \& Green, P. Maternal salivary levels of Streptococcus mutans and primary oral infection of infants. Arch Oral Biol 26, 147-149 (1981).

9. Funkhouser, L.J. \& Bordenstein, S.R. Mom knows best: the universality of maternal microbial transmission. PLoS biology 11, e1001631 (2013).

10. Dominguez-Bello, M.G., Costello, E.K., Contreras, M., Magris, M., Hidalgo, G., Fierer, N. \& Knight, R. Delivery mode shapes the acquisition and structure of the initial microbiota across multiple body habitats in newborns. Proceedings of the National Academy of Sciences of the United States of America 107, 11971-11975 (2010).

11. Cephas, K.D., Kim, J., Mathai, R.A., Barry, K.A., Dowd, S.E., Meline, B.S. \& Swanson, K.S. Comparative analysis of salivary bacterial microbiome diversity in edentulous infants and their mothers or primary care givers using pyrosequencing. PLoS ONE 6, e23503 (2011).

12. Song, S.J., Lauber, C., Costello, E.K., Lozupone, C.A., Humphrey, G., Berg-Lyons, D., Caporaso, J.G., Knights, D., Clemente, J.C., Nakielny, S., Gordon, J.I., Fierer, N. \& Knight, R. Cohabiting family members share microbiota with one another and with their dogs. Elife 2, e00458 (2013).

13. Crielaard, W., Zaura, E., Schuller, A.A., Huse, S.M., Montijn, R.C. \& Keijser, B.J. Exploring the oral microbiota of children at various developmental stages of their dentition in the relation to their oral health. BMC Med Genomics 4, 22 (2011).

14. Tanner, A.C., Milgrom, P.M., Kent, R., Jr., Mokeem, S.A., Page, R.C., Liao, S.I., Riedy, C.A. \& Bruss, J.B. Similarity of the oral microbiota of pre-school children with that of their caregivers in a population-based study. Oral microbiology and immunology 17 , 379-387 (2002). 
582 15. Caufield, P.W., Cutter, G.R. \& Dasanayake, A.P. Initial acquisition of mutans

583

584

585

586

587

588

589

590

591

592

593

594

595

596

597

598

599

600

601

602

603

604

605

606

607

608

609

610

611

612

613

614

615

616

617

618

619

620

621

622

623

624

625

626

627

streptococci by infants: evidence for a discrete window of infectivity. J Dent Res 72, 3745 (1993).

16. Wan, A.K., Seow, W.K., Walsh, L.J., Bird, P., Tudehope, D.L. \& Purdie, D.M. Association of Streptococcus mutans infection and oral developmental nodules in predentate infants. J Dent Res 80, 1945-1948 (2001).

17. Gurven, M., Kaplan, H. \& Supa, A.Z. Mortality experience of Tsimane Amerindians of Bolivia: regional variation and temporal trends. Am J Hum Biol 19, 376-398 (2007).

18. Martin, M., Blackwell, A., Gurven, M. \& Kaplan, H. in Primates, Pathogens, and Evolution. (eds. J. Brinkworth \& K. Pechenkina) 363-387 (Springer Science + Buisniness Media, New York; 2013).

19. Martin, M.A., Lassek, W.D., Gaulin, S.J., Evans, R.W., Woo, J.G., Geraghty, S.R., Davidson, B.S., Morrow, A.L., Kaplan, H.S. \& Gurven, M.D. Fatty acid composition in the mature milk of Bolivian forager-horticulturalists: controlled comparisons with a US sample. Matern Child Nutr 8, 404-418 (2012).

20. Veile, A., Martin, M., McAllister, L. \& Gurven, M. Modernization is associated with intensive breastfeeding patterns in the Bolivian Amazon. Soc Sci Med 100, 148-158 (2014).

21. Martin, M. (University of California Santa Barbara, 2015).

22. Schloss, P.D., Westcott, S.L., Ryabin, T., Hall, J.R., Hartmann, M., Hollister, E.B., Lesniewski, R.A., Oakley, B.B., Parks, D.H., Robinson, C.J., Sahl, J.W., Stres, B., Thallinger, G.G., Van Horn, D.J. \& Weber, C.F. Introducing mothur: open-source, platform-independent, community-supported software for describing and comparing microbial communities. Applied and environmental microbiology 75, 7537-7541 (2009).

23. Nilakanta, H., Drews, K.L., Firrell, S., Foulkes, M.A. \& Jablonski, K.A. A review of software for analyzing molecular sequences. BMC Res Notes 7, 830 (2014).

24. Schloss, P.D., Gevers, D. \& Westcott, S.L. Reducing the effects of PCR amplification and sequencing artifacts on 16S rRNA-based studies. PLoS ONE 6, e27310 (2011).

25. Paulson, J.N., Stine, O.C., Bravo, H.C. \& Pop, M. Differential abundance analysis for microbial marker-gene surveys. Nature methods 10, 1200-1202 (2013).

26. Yue, J.C., Clayton, M.K. \& Lin, F.C. A nonparametric estimator of species overlap. Biometrics 57, 743-749 (2001).

27. Lozupone, C., Lladser, M.E., Knights, D., Stombaugh, J. \& Knight, R. UniFrac: an effective distance metric for microbial community comparison. The ISME journal 5, 169172 (2011).

28. Anderson, M.J. A new method for non-parametric multivariate analysis of variance. Austral Ecology 26, 32-46 (2001).

29. Chen, T., Yu, W.H., Izard, J., Baranova, O.V., Lakshmanan, A. \& Dewhirst, F.E. The Human Oral Microbiome Database: a web accessible resource for investigating oral microbe taxonomic and genomic information. Database : the journal of biological databases and curation 2010, baq013 (2010).

30. Benjamini, Y.\& Hochberg, Y. Controlling the false discovery rate: a practical and powerful approach to multiple testing. Journal of the Royal Statistical Society Series B 57, 289-300 (1995).

31. Stahringer, S.S., Clemente, J.C., Corley, R.P., Hewitt, J., Knights, D., Walters, W.A., Knight, R. \& Krauter, K.S. Nurture trumps nature in a longitudinal survey of salivary 
628

629

630

631

632

633

634

635

636

637

638

639

640

641

642

643

644

645

646

647

648

649

650

651

652

653

654

655

656

657

658

659

660

661

662

663

664

665

666

667

668

669

670

671

672 bacterial communities in twins from early adolescence to early adulthood. Genome research 22, 2146-2152 (2012).

32. Zaura, E., Keijser, B.J., Huse, S.M. \& Crielaard, W. Defining the healthy "core microbiome" of oral microbial communities. BMC microbiology 9, 259 (2009).

33. Lazarevic, V., Gaia, N., Girard, M., Francois, P. \& Schrenzel, J. Comparison of DNA extraction methods in analysis of salivary bacterial communities. PLoS ONE 8, e67699 (2013).

34. Slots, J., Rams, T.E., Feik, D., Taveras, H.D. \& Gillespie, G.M. Subgingival microflora of advanced periodontitis in the Dominican Republic. J Periodontol 62, 543-547 (1991).

35. Cuesta, A.I., Jewtuchowicz, V., Brusca, M.I., Nastri, M.L. \& Rosa, A.C. Prevalence of Staphylococcus spp and Candida spp in the oral cavity and periodontal pockets of periodontal disease patients. Acta Odontol Latinoam 23, 20-26 (2010).

36. Palmer, C., Bik, E.M., DiGiulio, D.B., Relman, D.A. \& Brown, P.O. Development of the human infant intestinal microbiota. PLoS biology 5, e177 (2007).

37. Koenig, J.E., Spor, A., Scalfone, N., Fricker, A.D., Stombaugh, J., Knight, R., Angenent, L.T. \& Ley, R.E. Succession of microbial consortia in the developing infant gut microbiome. Proceedings of the National Academy of Sciences of the United States of America 108 Suppl 1, 4578-4585 (2011).

38. Yatsunenko, T., Rey, F.E., Manary, M.J., Trehan, I., Dominguez-Bello, M.G., Contreras, M., Magris, M., Hidalgo, G., Baldassano, R.N., Anokhin, A.P., Heath, A.C., Warner, B., Reeder, J., Kuczynski, J., Caporaso, J.G., Lozupone, C.A., Lauber, C., Clemente, J.C., Knights, D., Knight, R. \& Gordon, J.I. Human gut microbiome viewed across age and geography. Nature 486, 222-227 (2012).

39. Sampaio-Maia, B. \& Monteiro-Silva, F. Acquisition and maturation of oral microbiome throughout childhood: An update. Dent Res J (Isfahan) 11, 291-301 (2014).

40. Eribe, E.R. \& Olsen, I. Leptotrichia species in human infections. Anaerobe 14, 131-137 (2008).

41. Fujinaka, H., Takeshita, T., Sato, H., Yamamoto, T., Nakamura, J., Hase, T. \& Yamashita, Y. Relationship of periodontal clinical parameters with bacterial composition in human dental plaque. Archives of Microbiology 195, 371-383 (2013).

42. Jiang, W., Ling, Z., Lin, X., Chen, Y., Zhang, J., Yu, J., Xiang, C. \& Chen, H. Pyrosequencing Analysis of Oral Microbiota Shifting in Various Caries States in Childhood. Microbial ecology (2014).

43. Kort, R., Caspers, M., van de Graaf, A., van Egmond, W., Keijser, B. \& Roeselers, G. Shaping the oral microbiota through intimate kissing. Microbiome 2, 41 (2014).

44. Klein, M.I., Florio, F.M., Pereira, A.C., Hofling, J.F. \& Goncalves, R.B. Longitudinal study of transmission, diversity, and stability of Streptococcus mutans and Streptococcus sobrinus genotypes in Brazilian nursery children. Journal of Clinical Microbiology 42, 4620-4626 (2004).

45. Gizani, S., Papaioannou, W., Haffajee, A.D., Kavvadia, K., Quirynen, M. \& Papagiannoulis, L. Distribution of selected cariogenic bacteria in five different intra-oral habitats in young children. Int J Paediatr Dent 19, 193-200 (2009).

46. Tanner, A.C., Milgrom, P.M., Kent, R., Jr., Mokeem, S.A., Page, R.C., Riedy, C.A., Weinstein, P. \& Bruss, J. The microbiota of young children from tooth and tongue samples. J Dent Res 81, 53-57 (2002). 
673 47. Holgerson, P.L., Vestman, N.R., Claesson, R., Ohman, C., Domellof, M., Tanner, A.C.,

674

675

676

677

678

679

680

681

682

683

684

685

686

687

688

689

690

691

692

693

694

695

696

697

698

699

700

701

702

703

704

705

706
Hernell, O. \& Johansson, I. Oral microbial profile discriminates breast-fed from formulafed infants. J Pediatr Gastroenterol Nutr 56, 127-136 (2013).

48. Habicht, J.P. \& Pelto, G.H. Addressing epidemiological and public health analytic challenges in outcome and impact research: a commentary on 'Prechewing Infant Food, Consumption of Sweets and Dairy and Not Breastfeeding are Associated with Increased Diarrhea Risk of Ten Month Old Infants'. Matern Child Nutr 12, 625-631 (2016).

49. Cole, M.F., Evans, M., Fitzsimmons, S., Johnson, J., Pearce, C., Sheridan, M.J., Wientzen, R. \& Bowden, G. Pioneer oral streptococci produce immunoglobulin A1 protease. Infection and immunity 62, 2165-2168 (1994).

50. Al-Shehri, S.S., Knox, C.L., Liley, H.G., Cowley, D.M., Wright, J.R., Henman, M.G., Hewavitharana, A.K., Charles, B.G., Shaw, P.N., Sweeney, E.L. \& Duley, J.A. Breastmilk-Saliva Interactions Boost Innate Immunity by Regulating the Oral Microbiome in Early Infancy. PLoS ONE 10, e0135047 (2015).

51. Salone, L.R., Vann, W.F., Jr. \& Dee, D.L. Breastfeeding: an overview of oral and general health benefits. J Am Dent Assoc 144, 143-151 (2013).

52. White, V. Breastfeeding and the risk of early childhood caries. Evid Based Dent 9, 86-88 (2008).

53. Tham, R., Bowatte, G., Dharmage, S.C., Tan, D.J., Lau, M.X., Dai, X., Allen, K.J. \& Lodge, C.J. Breastfeeding and the risk of dental caries: a systematic review and metaanalysis. Acta Paediatr 104, 62-84 (2015).

54. Aas, J.A., Paster, B.J., Stokes, L.N., Olsen, I. \& Dewhirst, F.E. Defining the normal bacterial flora of the oral cavity. Journal of Clinical Microbiology 43, 5721-5732 (2005).

55. Gross, E.L., Beall, C.J., Kutsch, S.R., Firestone, N.D., Leys, E.J. \& Griffen, A.L. Beyond Streptococcus mutans: dental caries onset linked to multiple species by 16S rRNA community analysis. PLoS ONE 7, e47722 (2012).

56. Kanasi, E., Dewhirst, F.E., Chalmers, N.I., Kent, R., Jr., Moore, A., Hughes, C.V., Pradhan, N., Loo, C.Y. \& Tanner, A.C. Clonal analysis of the microbiota of severe early childhood caries. Caries research 44, 485-497 (2010).

57. Luo, A.H., Yang, D.Q., Xin, B.C., Paster, B.J. \& Qin, J. Microbial profiles in saliva from children with and without caries in mixed dentition. Oral Dis 18, 595-601 (2012). 
711 Figure 1. Relative abundance of the major bacterial phyla in mother and infant saliva samples. 712 Differences between groups of mother and infants for phyla Firmicutes and Fusobacteria are 713 significant $(* * p<0.01, * p<0.05)$.

Figure 2. Relative abundance of the major bacterial phyla of all saliva and premasticated food samples, sorted between infant and maternal subjects. BSA, infant saliva; MSA, mother saliva; FD, premasticated food; family index numbers are the same for infant, mother and food samples.

718

Figure 3. Two dimensional NMDS plot. Infant salivary microbiomes are closer to those of other infants than to their mothers in most cases. Round, infants; square, mothers. Stress $=0.054911$.

Figure 4. Average similarity distance of group of infants, mothers, related mother-infant dyads 723 and unrelated woman and infant pairs. ${ }^{* *}, \mathrm{p}<0.01$; ns, not significant, $\mathrm{p}>0.05$. 


\section{Suppl Figures}

725

726 Supplementary Figure 1. Rarefaction of 16S rRNA gene sequences of 24 saliva and two premasticated food samples.

Supplementary Figure 2. Average relative abundance of 15 genera reportedly enriched in cases

731

732

733 Supplementary Figure 3. Change of phylogenetic distance between mothers and infants. Average 734 dissimilarity distances between each infant and all mothers are negatively correlated with infant 735 age, $r=0.64, p=0.025$. Average dissimilarity distances between a mother and all infants are not 736 correlated with maternal age, $\mathrm{r}=-0.33, \mathrm{p}=0.293$.

737 